\section{COPD-Exazerbationen: Benralizumab bietet keinen Schutz}

Criner $\mathrm{Cl}$ et al. Benralizumab for the Prevention of COPD Exazerbations. NEJM 2019; doi:10.1056/NEJMoa1905248

Trotz einer Therapie mit zwei oder drei Pharmaka erleiden zahlreiche Patienten mit chronisch obstruktiver Lungenerkrankung (COPD) weiterhin Exazerbationen. Diese belastenden Ereignisse zu vermeiden, ist eins der wichtigsten Therapieziele. Da sich bei rund $40 \%$ der Betroffenen zudem eine eosinophile Entzündung nachweisen lässt, bietet sich Benralizumab, ein Anti-IL-5-Rezeptor-Antikörper, zur Prophylaxe an.

In zwei komplementären Phase-III-Studien (GALATHEA und TERRANOVA) war Benralizumab zur Therapie der mäßigen bis schweren COPD untersucht worden. In die Studien wurden Patienten in einem Alter von 40-85 Jahren (Exraucher und Raucher) mit mäßig bis sehr schwerer COPD aufgenommen, die trotz 2-Fachoder 3-Fach-Therapie mit Inhalativa bereits zwei therapiepflichtige Exazerbationen oder ein Ereignis mit erforderlicher stationärer Therapie erlitten hatten. Als Exazerbation galt eine mindestens 3-tägige Episode mit deutlicher Verschlechterung der Symptome, die eine systemische Therapie mit Kortison und/ oder Antibiotika oder eine stationäre Versorgung notwendig machte. Patienten mit einem Asthma bronchiale, das laut ärztlicher Einschätzung zur Symptomatik beitrug, wurden ausgeschlossen.

In den Studien wurden die Patienten randomisiert nach Grad der Eosinophilie ( $\geq 220$ vs. $<220 / \mathrm{mm}^{3}$ ); es ergab sich in etwa eine Ratio von 2:1. Die Dosierungen von Benralizumab lagen in der GALATHEA-Studie bei $30 \mathrm{mg}$ und $100 \mathrm{mg}$, in TERRANOVA bei $10 \mathrm{mg}, 30 \mathrm{mg}$ oder $100 \mathrm{mg}$. Die Gabe erfolgte als subkutane Injektion zunächst 4-wöchentlich, dann alle 8 Wochen über 13 Monate.
Da sich in vorherigen Studien ein Effekt bei COPD-Patienten mit Eosinophilie gezeigt hatte, gingen in die vorliegende Analyse nur Teilnehmer mit Werten für eosinophile Zellen von $\geq 220 / \mathrm{mm}^{3}$ ein. Der primäre Endpunkt - die errechnete Rate an Exazerbationen pro Jahr - ergab sich wie folgt:

- GALATHEA:

- Verum 30 mg: 1,19 Exazerbationen/Jahr (95\% Konfidenzintervall $[\mathrm{Cl}] 1,04-1,36)$,

- Verum 100 mg: 1,03 Exazerbationen/Jahr (95\%Cl 0,9-1,19),

- Placebo: 1,24 Exazerbationenen/ Jahr (95\% Cl 1,08-1,42).

- TERRANOVA:

- Verum $10 \mathrm{mg}: 0,99 / \mathrm{Jahr}$ (95\% Cl 0,87-1,13),

- Verum $30 \mathrm{mg}: 1,21 / \mathrm{Jahr}$ (95\% Cl 1,08-1,37)

- Verum $100 \mathrm{mg}: 1,09 / \mathrm{Jahr}$ $(95 \% \mathrm{Cl} 0,96-1,23)$

- Placebo: 1,17/Jahr (95\% Cl 1,04-1,32).

Aus diesen Daten ergaben sich Rate Ratios für GALATHEA von 0,96 (30 mg Benralizumab vs. Placebo) und 0,83 (100mg vs. Placebo) und für TERRANOVA von 0,85 (10 mg Verum), 1,04 (30 mg Verum) und 0,93 (100 mg Verum - jeweils vs. Placebo). Zum Zeitpunkt der Analyse nach 56 Wochen Therapie ließ sich für den 12-Monats-Zeitraum für keine der Dosierungen ein signifikanter Unterschied gegenüber Placebo zeigen. Anders sah es bei den eosinophilen Zellen und den respiratorischen Symptomen aus: Im Verlauf fielen die Werte für die Eosinophilen im Blut und im Sputum unter Benralizumab im Vergleich deutlich auf niedrige Werte und der GesamtScore im St. George's Respiratory Questionnaire (SGRQ) verbesserte sich im Vergleich zu Placebo. Unerwünschte Wirkungen traten in den Verum- und Placebo-Gruppen gleich häufig und in gleich verteilten Schweregraden auf.
FAZIT

Patienten mit mäßiger bis sehr schwerer COPD profitieren nicht von Benralizumab in verschiedenen Dosierungen bzgl. der Prävention von Exazerbationen. Der Antikörper führte zu einem signifikanten Abfall der eosinophilen Zellen, dieser Effekt führte jedoch nicht zu einer klinischen Besserung. Ob andere Biomarker sich eignen könnten, um die Patienten zu charakterisieren, bei denen ein Anti-IL5-Rezeptor-Antikörper wirksam ist, sollten weiter Studien zeigen, meinen die Autoren.

Dr. med. Susanne Meinrenken, Bremen 